\title{
Spatial Analysis and Mathematics in Health Research: Why
}

\author{
Pistolla Georgia $^{1}$, Mihael Maniadhakis ${ }^{2}$, Sifaki-Pistolla Dimitra ${ }^{1}$ \\ ${ }^{1}$ Faculty of Medicine, University of Crete, Iraklion, Greece \\ ${ }^{2}$ Faculty of Medicine, University of Crete, Athens, Greece
}

\section{Email address:}

geopist@gmail.com (G. Pistolla), spdimi11@gmail.com (D. Sifaki-Pistolla)

\section{To cite this article:}

Pistolla Georgia, Mihael Maniadhakis, Sifaki-Pistolla Dimitra. Spatial Analysis and Mathematics in Health Research: Why. Science Journal of Public Health. Special Issue: Spatial Analysis and Mathematics in Health Research, during Times of Global Socio-Economic Instability.

Vol. 3, No. 3-1, 2015, pp. 1-3. doi: 10.11648/j.sjph.s.2015030301.11

\begin{abstract}
The present report is a short introduction of the special issue entitled "Spatial Analysis and Mathematics in Health Research, during Times of Global Socio-Economic Instability". It aims to discuss several issues regarding the methodologies and analytics in health research as well as to raise questions and give "food for thought" about the way researchers manage health data. Finally, it outlines the use of mathematics and spatio-temporal analysis in research in epidemiology, Public health, Primary health care and health care practice.
\end{abstract}

Keywords: Health Principles, Spatial Analysis, Deterministic, Stochastic, GIS

\section{Short Introduction}

Innovative methodologies and inter-sector collaboration are the key points of all discussion forums for health research, well-being and healthy ageing, globally. At the same time, conventional methodologies or data management approaches that are commonly used in health research, are often questioned nowadays. This is mainly due to the fact that health policies and public health strategies haven't yet shown the expected results.

The present report is a short introduction of the special issue entitled "Spatial Analysis and Mathematics in Health Research, during Times of Global Socio-Economic Instability", that aims to discuss several issues regarding the methodologies and analytics in health research as well as to raise questions and give "food for thought" about the way researchers manage health data.

\section{Reflections on Methodological Issues}

We should start with two major facts that are widely accepted from the scientific community:

a) The basic epidemiological principles are person-spacetime.

b) Organs of human body (e.g. lungs or breast) as well as their functions are fractal

Based on the epidemiological principles and the biology of the human body, health-related data should be analyzed and managed in three dimensions or based on both quantitative and qualitative components. The most reasonable question would be the following: Why the result of human organs' functions or the impact that they receive from external factors, should be considered as stochastic (which refers to linear methods of analysis), unconditionally?

The first step for every health researcher or statistician should be to set several questions before going on with the analysis and try to answer them in order to develop a valid and of high quality methodological framework. For instance, "Are my data stochastic or deterministic?" (both for spatial or longitudinal data) [1-3]. The answer to this question would provide us with reliable ways of data analysis by using statistical or mathematical methods. There are a few studies and results that prove this and often surprise the researchers. Such results were exported from Greek data (number of deaths and mortality rates) and revealed interactions of a deterministic nature [2] which lead to non-linear methods of analysis [3]. This revealed the need of extensive research over various health rates and data even from other countries, in order to test their nature and patterns (eg. if they are deterministic or stochastic and whether they follow similar patterns).

The second step should be to develop an analysis "environment" for health-related data, as close to their real environment as it is possible. Spatial analysis allows us to study all types of phenomena as closer as to their real 
environment based on their three dimensions ( $\mathrm{x} ; \mathrm{y}$ coordinates and time). As it is outlined in various papers and specifically in the articles of Van $\mathrm{H}$ et al. and Krieger N. [4,5] mortality and morbidity varies among different regions and time units, even when we speak about the same city or neighboring towns. Van $\mathrm{H}$ et al. used spatio-temporal regression models in their study, while Krieger N. emphasized on the use of such type of analyses and the fact that they are vital for Health research [5]. This proves that the philosophy of epidemiology, medicine and these methods of analysis have a common start point and are "built" over the three pre-mentioned principles: person, time, space.

Particularly, spatial statistics and dynamic mathematical methods of analysis are a leading methodological framework that works towards that direction. Spatial models and procedures are examined to lead to a conversation regarding policies in the Health area and Health management, as well as the study of various epidemiological issues [6,7]. They are already recognized as an integral ingredient in health research in order to develop effective policies for Public health and health care practice. Geographical Information Systems (GIS) or other mathematical models and algorithms are able to examine the spatial and temporal dynamics of diseases or health-related outcomes. A range of themes and topics need to be studied under the light of these approached; for instance positive health outcomes such as well-being, "good" health, healthy ageing, prevention of chronic diseases, actions for policy development and community-based interventions, innovative clinical research with an impact on personalized care.

Results of cluster and spatial analysis in a Greek study depict a grouping of Prefectures that are of similar behavior according to mortality rates and socioeconomic factors. Through the application of an inventive algorithm and a totally new theory (Spatial Structure Function), it was proved that the behavior of each Prefecture cannot be grouped according to administrative limits [2,5]. Prefectures that weren't spatially proximate, are often clustered together. For instance, Pr. of Iraklion, Pr. of Chania and Pr. of Dodekanisos were in the same cluster with Pr. of Evros or other Prefectures of Northern or central Greece. High mortality rates had been detected at the area of Attiki (13.59), Evros (9.7), Grevena (9.5) and Trikala (9.2). On the other hand, lower rates have been presented in Pr. of Kerkyra (2.6), Samos (2.6) and Kyklades (2.7) [2]. Therefore, Health strategies that are based on administrative limits or on generalized national plans would be ineffective in reducing negative health outcomes and promote health, in regions that present special behavioral patterns. Unfortunately, in many countries as well as in Greece, policies are structured at a national and general level with no special focus on high risk areas and their quality characteristics. This is a phenomenon that widens during periods of economic and social crisis and has an effect on the expansion of health and social inequalities.

\section{3. "Food for Thought"}

The topics raised in this special issue derived from a direct need to estimate causative relations factors and identify the factors that cause the heterogeneity of disease's distribution among the different regions and population groups. These factors could be environmental, social, economical, cultural, genetic or behavioral factors [5,7]. According to the literature, most of the studies include relevant information and data, yet they are approached using conventional statistics (1 dimensional approach) rather than spatial statistics (multidimensional approach) $[8,9]$.

Two interesting examples that support the other side of the coin, are the following: the USA that has adapted an approach based on the GIS technology in health research and Australia that recently developed and adapted an integrated GIS tool in all national topics (health, tourism, law, business, familyhome-community, defense, culture, transportation etc) [10-13]. So, why shouldn't we try to "taste" the results of a different approach?

\section{References}

[1] Jonathan B, Dingwell, Joseph P, Cusuman O. Nonlinear time series analysis of normal and pathological human walking. Chaos 2000; 10: 96-101.

[2] Pistolla G, Prastakos P, Vassilaki M, Philalithis A. Spatialmathematic methods for analysis of indicators of mortality. International journal of advanced engineering sciences and technologies 2011; 10: 136-137.

[3] Sugihara G, May R. Nonlinear forecasting as a way of distinguishing chaos from measurement error in time series. Nature 1990; 344: 734-740.

[4] Van H, Victor CS, Adam AP, Ronaldd N, Jonathane W, Karole, Davida KP, Sungg K, Joel D. Association of Long-term Air Pollution With Ventricular Conduction and Repolarization Abnormalities. Epidemiology J 2011; 22: 773-780.

[5] Krieger N. Place, Space, and Health: GIS and Epidemiology. Epidemiology J 2003; 14: 384-385

[6] Matthews SA. Epidemiology using a GIS: the need for caution. Comput Environ Urban Syst 1990; 14:213-21.

[7] Mayer JD. The role of spatial analysis and geographic data in the detection of disease causation. Soc Sci Med1983;17:121321

[8] Llongueras S, Kogevinas M, Roman E, Regional Differences in Maternal Mortality in Greece, 1973-1982. Int. J. Epidemiol. 1988; 17: 574-578.

[9] Christopher JLM , Lopez AD. Mortality by cause for eight regions of the world: Global Burden of Disease Study. The Lancet 1997 ; 349: 1269 - 1276.

[10] Hurley S, Saunders T, Nivas R, Hertz A, Reynolds P. Post office box addresses: a challenge for geographic information system-based studies. Epidemiology. 2003; 14: 386-391. 
[11] McElroy J, Remington P, Trentham-Dietz A, Robert S, Newcomb P. Geocoding addresses from a large populationbased study: lessons learned. Epidemiology. 2003; 14: 399-407.

[12] Floret N, Mauny F, Challier B, Arveux P, Arveux J-Y, Viel J-F. Dioxin emissions from a solid waste incinerator and risk of non-Hodgkin's lymphoma. Epidemiology. 2003; 14: 392-398.
$[13$ GIS. Australia government. Av
http://www.australia.gov.au/topics/science-and-
technology/spatial-information. http://www.australia.gov.au/topics/science-andAvailable at: 